\title{
Chronological change of gene expression during the transdifferentiation from human amniotic epithelial cells to pancreatic endocrine cells in vitro
}

\author{
Jian Wang ${ }^{1, *}$, Lin Peng ${ }^{1, *}$, Xiao Liu ${ }^{1}$, Guangxiu Lu ${ }^{1}$ \\ ${ }^{1}$ National Center of Human Stem Cell Research and Engineering, Institute of Human Reproduction and Stem Cell Engineering, \\ Central South University, Changsha, Hunan 410078, China
}

Studies on the HAEC (human amniotic epithelial cell), a kind of stem cells, have suggested that it could be an excellent resource for cell replacement in the clinical practice, once differentiated into functional cells. In 20042005, transplantation of HAECs derived insulin secreting cells, induced by the nicotinamide and $\mathrm{N} 2$ in vitro, into streptozotocin-induce diabetic mice normalized the hyperglycemia. However, these cells only sustained the normal blood glucose for only tow months and could express both insulin and glucagon genes according to recent report, seemed that they were not stable and mature insulin secreting cells.

To get a better understanding of the transdifferentiation characteristics, we evaluated the chronological gene expression changes, such as PDX1, ngn3, pax4, pax6, nkx6.1, nkx2.2, insulin and glucose gene, by semi-quantity RT-PCR during a 14-day inductive course. Briefly, the cell samples were collected every other day and then semiquantity RT-PCR was performed to examine the mRNA expression level. The immunocytofluorescental analysis were performed to detect the insulin or/and glucagon-positive cell number changes. The gene chronological expression analysis demonstrated a different expression trend between insulin and glucagon gene. Insulin gene's expression was first up-regulated in the first week and subsequently down-regulated, but the glucagon was sustained at a stable high level within the whole time course. Expression tendency of PDX1, pax4, nkx2.2 gene, known as important transcriptional factor for beta cell differentiation and insulin gene transcription, was similar to that of insulin gene. PDX1 was even negative in the last 4 days. Ngn3, nkx6.1 and pax6, which was important for alpha cell differentiation, were stable expressed in all of the samples except HAECs. Immunocytofluorescent analysis showed that double positive cells were the major part in the first week, after that, number of glucagons-positive cells increased step by step, whereas insulin-positive cells number decreased. Our results indicated that prevalent inductive method of HAECs-derived insulin secreting cells by addition of $\mathrm{N} 2$ and nicotinamide was not effective enough and many HAECs differentiated into glucagon secreting cells.

Keywords: human amniotic epithelial cells, transdifferentiation, insulin secreting cell

Cell Research (2008) 18:s158. doi: 10.1038/cr.2008.248; published online 4 August 2008

*These two authors contributed equally to this work.

Correspondence: Guangxiu Lu

E-mail: lgxdirector@yahoo.com.cn 